\title{
Delocalization of wave packets in disordered nonlinear chains
}

\author{
Ch. Skokos, ${ }^{1}$ D. O. Krimer, ${ }^{1}$ S. Komineas,${ }^{2}$ and S. Flach ${ }^{1}$ \\ ${ }^{1}$ Max Planck Institute for the Physics of Complex Systems, \\ Nöthnitzer Str. 38, D-01187 Dresden, Germany \\ ${ }^{2}$ Department of Applied Mathematics, \\ University of Crete, GR-71409 Heraklion, Crete, Greece
}

(Dated: August 22, 2018)

\begin{abstract}
We consider the spatiotemporal evolution of a wave packet in disordered nonlinear Schrödinger and anharmonic oscillator chains. In the absence of nonlinearity all eigenstates are spatially localized with an upper bound on the localization length (Anderson localization). Nonlinear terms in the equations of motion destroy Anderson localization due to nonintegrability and deterministic chaos. At least a finite part of an initially localized wave packet will subdiffusively spread without limits. We analyze the details of this spreading process. We compare the evolution of single site, single mode and general finite size excitations, and study the statistics of detrapping times. We investigate the properties of mode-mode resonances, which are responsible for the incoherent delocalization process.
\end{abstract}

PACS numbers: 05.45.-a, 05.60.Cd, 63.20.Pw 


\section{INTRODUCTION}

The normal modes (NMs) of a $d=1$-dimensional linear system with uncorrelated random potential are spatially localized (Anderson localization). Therefore any wave packet, which is initially localized, remains localized for all time [1]. Note that NMs correspond to single particle eigenstates of related quantum systems.

When nonlinearities are added, NMs interact with each other [2]. Recently, experiments were performed on light propagation in spatially random nonlinear optical media [3, 4] and on Bose-Einstein condensate expansions in random optical potentials [5], which serve as realizations of such cases.

Numerical studies of wave packet propagation in several models showed that the second moment of the norm/energy distribution grows subdiffusively in time as $t^{\alpha}[\underline{6}$ ], with $\alpha \approx$ $1 / 3$ for $d=1$. Reports on partial localization were published as well [10].

In a recent letter the mechanisms of spreading and localization were studied for $d=1$, with initial excitations being localized on a single site [11]. A theoretical explanation of the exponent $\alpha=1 / 3$ was obtained, consistently assuming that the internal dynamics of a wave packet is chaotic, leading to a partial dephasing of the NMs. The argumentation was based on the possibility of a pair of wave packet modes being able to resonantly interact with each other. Among other results, the case of weak nonlinearity showed that wave packets localize according to the linear dynamics on long but finite time scales, with subsequent detrapping. In the present work, we extend this study to single mode excitations, and more general excitations of width $L$. We study the details of the detrapping process, and measure the statistical properties of detrapping times. We study the particularities of resonant interaction between modes, mediated by the nonlinearity. We give details on the used integration schemes, and perform extensive tests which demonstrate that the observed effects are not affected by roundoff errors. We argue that the spreading is inherently induced by the nonintegrability of the system.

\section{MODELS}

We study two models of one-dimensional lattices. 


\section{A. Nonlinear Schrödinger lattice}

The Hamiltonian of the disordered discrete nonlinear Schrödinger equation (DNLS)

$$
\mathcal{H}_{D}=\sum_{l} \epsilon_{l}\left|\psi_{l}\right|^{2}+\frac{\beta}{2}\left|\psi_{l}\right|^{4}-\left(\psi_{l+1} \psi_{l}^{*}+\psi_{l+1}^{*} \psi_{l}\right)
$$

with complex variables $\psi_{l}$, lattice site indices $l$ and nonlinearity strength $\beta \geq 0$. The random on-site energies $\epsilon_{l}$ are chosen uniformly from the interval $\left[-\frac{W}{2}, \frac{W}{2}\right]$, with $W$ denoting the disorder strength. The equations of motion are generated by $\dot{\psi}_{l}=\partial \mathcal{H}_{D} / \partial\left(i \psi_{l}^{\star}\right)$ :

$$
i \dot{\psi}_{l}=\epsilon_{l} \psi_{l}+\beta\left|\psi_{l}\right|^{2} \psi_{l}-\psi_{l+1}-\psi_{l-1}
$$

Eqs. (21) conserve the energy (1) and the norm $S=\sum_{l}\left|\psi_{l}\right|^{2}$. We note that varying the norm of an initial wave packet is strictly equivalent to varying $\beta$, therefore we choose $S=1$. Eqs. (1) and (2) are derived e. g. when describing two-body interactions in ultracold atomic gases on an optical lattice within a mean field approximation [12], but also when describing the propagation of light through networks of coupled optical waveguides in Kerr media [13].

For $\beta=0$ Eq. (1) with $\psi_{l}=A_{l} \exp (-i \lambda t)$ is reduced to the linear eigenvalue problem

$$
\lambda A_{l}=\epsilon_{l} A_{l}-A_{l-1}-A_{l+1} .
$$

The normalized eigenvectors $A_{\nu, l}\left(\sum_{l} A_{\nu, l}^{2}=1\right)$ are the NMs, and the eigenvalues $\lambda_{\nu}$ are the frequencies of the NMs. The width of the eigenfrequency spectrum $\lambda_{\nu}$ of (3) is $\Delta_{D}=W+4$ with $\lambda_{\nu} \in\left[-2-\frac{W}{2}, 2+\frac{W}{2}\right]$.

The asymptotic spatial decay of an eigenvector is given by $A_{\nu, l} \sim \mathrm{e}^{-l / \xi\left(\lambda_{\nu}\right)}$ where $\xi\left(\lambda_{\nu}\right) \leq \xi(0) \approx 100 / W^{2}$ is the localization length [14]. The NM participation number $p_{\nu}=1 / \sum_{l} A_{\nu, l}^{4}$ characterizes the spatial extend (localization volume) of the NM. It is distributed around the mean value $\overline{p_{\nu}} \approx 3.6 \xi\left(\lambda_{\nu}\right)$ with variance $\approx 1.3 \xi\left(\lambda_{\nu}\right)$ [15]. The average spacing of eigenvalues of NMs within the range of a localization volume is therefore $\overline{\Delta \lambda}_{D} \approx \Delta_{D} / \overline{p_{\nu}} \approx \Delta_{D} W^{2} / 360$. The two scales $\overline{\Delta \lambda}_{D} \leq \Delta_{D}$ determine the packet evolution details in the presence of nonlinearity.

The equations of motion of (1) in normal mode space read

$$
i \dot{\phi}_{\nu}=\lambda_{\nu} \phi_{\nu}+\beta \sum_{\nu_{1}, \nu_{2}, \nu_{3}} I_{\nu, \nu_{1}, \nu_{2}, \nu_{3}} \phi_{\nu_{1}}^{*} \phi_{\nu_{2}} \phi_{\nu_{3}}
$$


with the overlap integral

$$
I_{\nu, \nu_{1}, \nu_{2}, \nu_{3}}=\sum_{l} A_{\nu, l} A_{\nu_{1}, l} A_{\nu_{2}, l} A_{\nu_{3}, l}
$$

The variables $\phi_{\nu}$ determine the complex time-dependent amplitudes of the NMs.

The frequency shift of a single site oscillator induced by the nonlinearity is $\delta_{l}=\beta\left|\psi_{l}\right|^{2}$. If instead a single mode is excited, its frequency shift is given by $\delta_{\nu}=\beta\left|\phi_{\nu}\right|^{2} / p_{\nu}$.

\section{B. Anharmonic oscillator lattice}

The Hamiltonian of the quartic Klein-Gordon lattice (KG)

$$
\mathcal{H}_{K}=\sum_{l} \frac{p_{l}^{2}}{2}+\frac{\tilde{\epsilon}_{l}}{2} u_{l}^{2}+\frac{1}{4} u_{l}^{4}+\frac{1}{2 W}\left(u_{l+1}-u_{l}\right)^{2},
$$

where $u_{l}$ and $p_{l}$ are respectively the generalized coordinates and momenta, and $\tilde{\epsilon}_{l}$ are chosen uniformly from the interval $\left[\frac{1}{2}, \frac{3}{2}\right]$. The equations of motion are $\ddot{u}_{l}=-\partial \mathcal{H}_{K} / \partial u_{l}$ and yield

$$
\ddot{u}_{l}=-\tilde{\epsilon}_{l} u_{l}-u_{l}^{3}+\frac{1}{W}\left(u_{l+1}+u_{l-1}-2 u_{l}\right) .
$$

Equations (7) conserve the energy (6). They serve e.g. as simple models for the dissipationless dynamics of anharmonic optical lattice vibrations in molecular crystals [16]. The energy of an initial state $E \geq 0$ serves as a control parameter of nonlinearity similar to $\beta$ for the DNLS case.

The coefficient $1 /(2 W)$ in ([6) was chosen so that the linear parts of Hamiltonians (1) and (6) would correspond to the same eigenvalue problem. In practice, for $E \rightarrow 0$ (or by neglecting the nonlinear term $\left.u_{l}^{4} / 4\right)$ model (6) with $u_{l}=A_{l} \exp (i \omega t)$ is reduced to the linear eigenvalue problem (3) with $\lambda=W \omega^{2}-W-2$ and $\epsilon_{l}=W\left(\tilde{\epsilon}_{l}-1\right)$. The width of the squared

frequency $\omega_{\nu}^{2}$ spectrum is $\Delta_{K}=1+\frac{4}{W}$ with $\omega_{\nu}^{2} \in\left[\frac{1}{2}, \frac{3}{2}+\frac{4}{W}\right]$. Note that $\Delta_{D}=W \Delta_{K}$. As in the case of DNLS, $W$ determines the disorder strength.

The spatial properties of the NMs are identical with those of (3). In addition to the scale $\Delta_{K}$, the average spacing of squared eigenfrequencies of NMs within the range of a localization volume is $\overline{\Delta \omega^{2}}=\Delta_{K} / \overline{p_{\nu}}$. The two scales $\overline{\Delta \omega^{2}} \leq \Delta_{K}$ determine the packet evolution details in the presence of nonlinearity.

The squared frequency shift of a single site oscillator induced by the nonlinearity is $\delta_{l} \approx\left(3 E_{l}\right) /\left(2 \tilde{\epsilon}_{l}\right)$, where $E_{l}$ is the energy of the oscillator. If instead a single mode is excited, its frequency shift is given by $\delta_{\nu} \approx\left(3 E_{\nu}\right) /\left(2 p_{\nu} \omega_{\nu}^{2}\right)$ with $E_{\nu}$ being the energy of the mode. 
For small amplitudes the equations of motion of the $\mathrm{KG}$ chain can be approximately mapped onto a corresponding DNLS model [17]. In our notation, the mapping takes the following form. For the KG model with given parameters $W$ and $E$, the corresponding DNLS model (11) with norm $S=1$, has a nonlinearity parameter $\beta \approx 3 W E$. The norm density of the DNLS model corresponds to the normalized energy density of the KG model.

\section{Computational methods}

We will present results on long time numerical simulations. We therefore first discuss the methods and particularities of our computations. For both models, we used symplectic integrators. These integration schemes replace the original Hamiltonian by a slightly different one, which is integrated exactly. The smaller the time steps, the closer both Hamiltonians. Therefore, the computed energy (or norm) of the original Hamiltonian function will fluctuate in time, but not grow. The fluctuations are bounded, and are due to the fact, that the actual Hamiltonian which is integrated, has slightly different energy.

Another possible source of errors is the roundoff procedure of the actual processor, when performing operations with numbers. Sometimes it is referred to as 'computational noise' although it is exactly the opposite, i. e. purely deterministic and reproducible. We will discuss the influence of roundoff errors on our results in section

The KG chain was integrated with the help of a symplectic integrator of order $\mathcal{O}\left(\tau^{4}\right)$ with respect to the integration time step $\tau$, namely the $\mathrm{SABA}_{2}$ integrator with corrector $\left(\mathrm{SABA}_{2} \mathrm{C}\right)$, introduced in [18]. A brief presentation of the integration scheme, as well as its implementation for the particular case of the KG lattice (6) is given in Appendix A. The $\mathrm{SABA}_{2} \mathrm{C}$ integration scheme proved to be very efficient for long integrations (e. g. up to $10^{10}$ time units) of lattices having typically $N=1000$ sites (see for example the right plots of Fig. 21), since it kept the required computational time to feasible levels, preserving at the same time quite well the energy of the system. For example, an integration time step $\tau=0.2$ usually kept the relative error of the energy smaller than $10^{-4}$.

The DNLS chain was integrated with the help of the $\mathrm{SBAB}_{2}$ integrator (see Appendix A), which introduces an error in energy conservation of the order $\mathcal{O}\left(\tau^{2}\right)$. The number of sites used in our computations varied from $N=500$ to $N=2000$, in order to exclude finite size effects in the evolution of the wave packets. For $\tau=0.1$ the relative error of energy was 
usually kept smaller than $10^{-3}$. It is worth mentioning that, although the $\mathrm{SBAB}_{2}$ integrator and the commonly used leap-frog integrator introduce errors of the same order, the $\mathrm{SBAB}_{2}$ scheme exhibits a better performance since it requires less CPU time, keeping at the same time the relative energy error to smaller values than the leap-frog scheme.

We order the NMs in space by increasing value of the center-of-norm coordinate $X_{\nu}=$ $\sum_{l} l A_{\nu, l}^{2}$. We analyze normalized distributions $z_{\nu} \geq 0$ using the second moment $m_{2}=$ $\sum_{\nu}(\nu-\bar{\nu})^{2} z_{\nu}$, which quantifies the wave packet's degree of spreading and the participation number $P=1 / \sum_{\nu} z_{\nu}^{2}$, which measures the number of the strongest excited sites in $z_{\nu}$. Here $\bar{\nu}=\sum_{\nu} \nu z_{\nu}$. For DNLS we follow norm density distributions $z_{\nu} \equiv\left|\phi_{\nu}\right|^{2} / \sum_{\mu}\left|\phi_{\mu}\right|^{2}$. For KG we follow normalized energy density distributions $z_{\nu} \equiv E_{\nu} / \sum_{\mu} E_{\mu}$ with $E_{\nu}=\dot{A}_{\nu}^{2} / 2+\omega_{\nu}^{2} A_{\nu}^{2} / 2$, where $A_{\nu}$ is the amplitude of the $\nu$ th NM and $\omega_{\nu}^{2}=1+\left(\lambda_{\nu}+2\right) / W$.

\section{WAVE PACKET EVOLUTION}

Below we will mainly use the DNLS case for theoretical considerations, and also discuss crucial points to be taken into account, when considering the KG case. We will present numerical results for both models.

We first consider a wave packet at $t=0$ which is compact either in real space, or in normal mode space. Compactness in real space implies a single site excitation $\psi_{l}=\delta_{l, l_{0}}$ with the choice $\epsilon_{l_{0}}=0$ for the DNLS model. For the KG model we set $p_{l}=0, u_{l}=c \delta_{l, l_{0}}$ with $\tilde{\epsilon}_{l_{0}}=1$ and $c$ being a constant which defines the initial energy $E$. Compactness in normal mode space instead implies a single mode excitation $\phi_{\nu}=\delta_{\nu, \nu_{0}}$ with $\lambda_{\nu_{0}} \approx 0$ for the DNLS model, while in the case of the KG system we have $A_{\nu}=c \delta_{\nu, \nu_{0}}, \dot{A}_{\nu}=0$, with $\omega_{\nu_{0}}^{2} \approx 1+(2 / W)$, i. e. $\omega_{\nu_{0}}^{2}$ is located in the middle of the frequency spectrum. Again the constant $c$ defines the initial energy of the wave packet. We will later also consider finite size initial distributions of width $L$.

\section{A. Expected regimes}

Let us consider a single site initial excitation with a corresponding nonlinear frequency shift $\delta_{l}$. We compare this frequency shift with the two scales set by the linear equations: the average spacing $\overline{\Delta \lambda}$ (which corresponds to $\overline{\Delta \lambda}_{D}$ for DNLS and to $\overline{\Delta \omega^{2}}$ for KG) and 


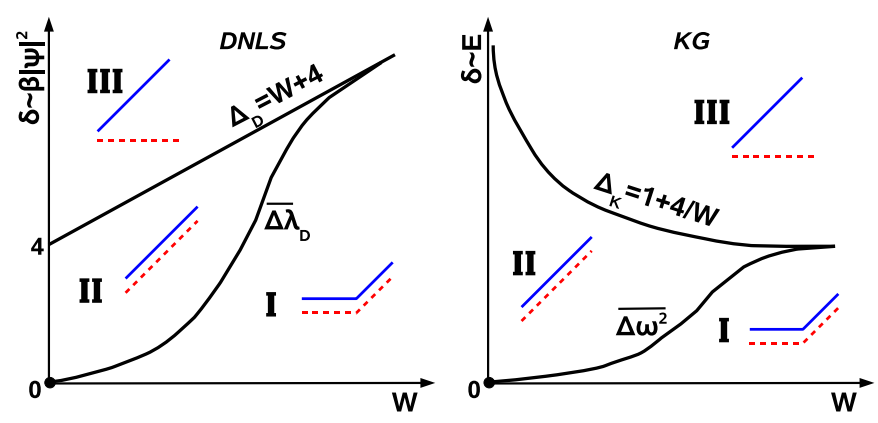

FIG. 1: (color online) Schematic representations of the three different regimes of spreading for the DNLS (left graph) and the KG model (right graph), in the parameter space of disorder strength $W$ and of the nonlinear frequency shift $\delta$ at initial time $t=0$. For each regime the dependence of $\log m_{2}$ (blue solid curves) and of $\log P$ (red dashed curves) versus $\log t$ are shown schematically (see section IIIC for details).

the spectrum width $\Delta$ (with $\Delta$ denoting $\Delta_{D}$ for DNLS and $\Delta_{K}$ for KG). We expect three qualitatively different dynamical regimes: I) $\delta_{l}<\overline{\Delta \lambda}$; II) $\overline{\Delta \lambda}<\delta_{l}<\Delta$; III) $\Delta<\delta_{l}$. In case I the local frequency shift is less than the average spacing between interacting modes, therefore no initial resonance overlap of them is expected, and the dynamics may (at least for long times) evolve as in the linear case ( $\beta=0$ for DNLS and $E \rightarrow 0$ for KG). In case II resonance overlap may happen immediately, and the packet should evolve differently. For case III the frequency shift exceeds the spectrum width, therefore some renormalized frequencies of NMs (or sites) may be tuned out of resonance with the NM spectrum, leading to selftrapping. The above definitions are highly qualitative, since localized initial conditions are subject to strong fluctuations.

If we instead consider a single mode initial excitation, we have to replace $\delta_{l}$ by $\delta_{\nu}$ in the above argumentation. For both the DNLS and the KG model, it follows $\delta_{l} \sim p_{\nu} \delta_{\nu}$. The mean NM participation number (the localization volume) $\overline{p_{\nu}}>1$ depends on the disorder strength $W$.

If an initial excitation of the DNLS model is characterized by some exponentially localized (not necessarily compact) distribution $\psi_{l}$ with $S=1$, the nonlinear frequency shift may be roughly estimated as $\delta \sim \beta|\psi|^{2}$, where the maximum norm density $|\psi|^{2}=\sup _{l}\left|\psi_{l}\right|^{2}$. The left graph of Fig.1 shows the location of the three different regimes in the plane of the control parameters, i. e. the frequency shift $\delta$ and the disorder strength $W$. Note that $\overline{\Delta \lambda} \propto W^{3}$ 
for $W \ll 1$ [15], and the intermediate regime II disappears around $W \approx 20$, where the participation number of a NM becomes of the order of one, and the NMs become almost single site solutions. Similarly, for the KG model we have the estimation $\delta \sim E$ and the corresponding parameter space of the three different regimes is shown in the right graph of Fig:1,

\section{B. The selftrapping theorem}

Regime III is also captured by a theorem presented in [10], which proves, that for $\beta>\Delta$ (for the DNLS case) the single site excitation can not uniformly spread over the entire (infinite) lattice. Indeed, with the notations

$$
\begin{array}{r}
\mathcal{H}_{D}=\mathcal{H}_{N L}+\mathcal{H}_{L} \\
\mathcal{H}_{L}=\sum_{l} \epsilon_{l}\left|\psi_{l}\right|^{2}-\left(\psi_{l+1} \psi_{l}^{*}+\psi_{l+1}^{*} \psi_{l}\right) \\
\mathcal{H}_{N L}=\sum_{l} \frac{\beta}{2}\left|\psi_{l}\right|^{4} \equiv \frac{\beta}{2} P_{r}^{-1}
\end{array}
$$

where $P_{r}$ is the participation number in real space, the single site excitation at time $t=0$ yields

$$
\mathcal{H}_{L}(t=0)=0, \mathcal{H}_{N L}(t=0)=\frac{\beta}{2} .
$$

Due to norm conservation $S=1$ at all times, the harmonic energy part $\mathcal{H}_{L}$ is bounded from above and below [10]:

$$
-2-\frac{W}{2} \leq \mathcal{H}_{L} \leq 2+\frac{W}{2}
$$

Due to energy conservation, for all times the anharmonic energy part $\mathcal{H}_{N L}$ can therefore not become smaller than

$$
\mathcal{H}_{N L}(t) \geq \frac{\beta}{2}-2-\frac{W}{2}
$$

It follows with (10), that the participation number is bounded from above by a finite number, which diverges for $\beta=\Delta$ :

$$
P_{r}(t) \leq \frac{\beta}{\beta-\Delta} \text { if } \beta \geq \Delta .
$$

Moreover, since $P_{r}^{-1}=\sum_{l}\left|\psi_{l}\right|^{4}<\sup _{l}\left|\psi_{l}\right|^{2}[10]$, we conclude that

$$
\sup _{l}\left|\psi_{l}\right|^{2}(t)>\frac{\beta-\Delta}{\beta} \text {. }
$$


Therefore, at least a part of the wave packet will not spread, and stay localized, although the theorem does not prove that the location of that inhomogeneity is constant in time. The norm of the part of the wave packet, which can spread uniformly over the entire system, is bounded from above by $S_{\infty} \leq \Delta / \beta$.

\section{Numerical results}

We first show results for single site excitations [11]. We systematically studied the evolution of wave packets for lattices (1) and (6). The scenario described in section [IIA was observed very clearly. Representative examples are shown in Fig,2, Regime III yields selftrapping (see also Figs. 1, 3 in [10]), therefore $P$ does not grow significantly, while the second moment $m_{2} \sim t^{\alpha}$ with $\alpha \approx 1 / 3$ (red curves). Thus a part of the excitation stays highly localized [10], while another part delocalizes. Regime II yields subdiffusive spreading with $m_{2} \sim t^{\alpha}$ and $P \sim t^{\alpha / 2}$ [7, 8] (green curves). Regime I shows Anderson localization up to some time $\tau_{d}$ which increases with decreasing nonlinearity. For $t<\tau_{d}$ both $m_{2}$ and $P$ are not changing. However for $t>\tau_{d}$ a detrapping takes place, and the packet starts to grow with characteristics as in regime II (blue curves). The simulation of the equations of motion in the absence of nonlinear terms (orange curves), demonstrates the appearance of Anderson localization.

The second moment $m_{2}$ is sensitive to the spreading distance of the tails of a distribution, while the participation number $P$ is a measure of the inhomogeneity of the distribution, being insensitive to any spatial correlations. Thus, $P$ and $m_{2}$ can be used to quantify the sparseness of a wave packet. To this end, we introduce as a measure of the compactness of a wave packet the compactness index

$$
\zeta=\frac{P^{2}}{m_{2}} .
$$

Let us consider a wave packet of $K$ sites $(K \gg 1)$. In the case where all the $K$ sites are equally excited the compactness index is given by $\zeta=12$. In the case of a symmetric wave packet formed by a sequence of an excited site followed by a nonexcited one, where all the $K / 2$ excited sites have the same amplitude, $\zeta=3$. Distributions with larger gaps between the equally excited isolated sites attain a compactness index $\zeta<3$. For the extreme case of a sparse wave packet formed by two equally excited sites located at the two edges 


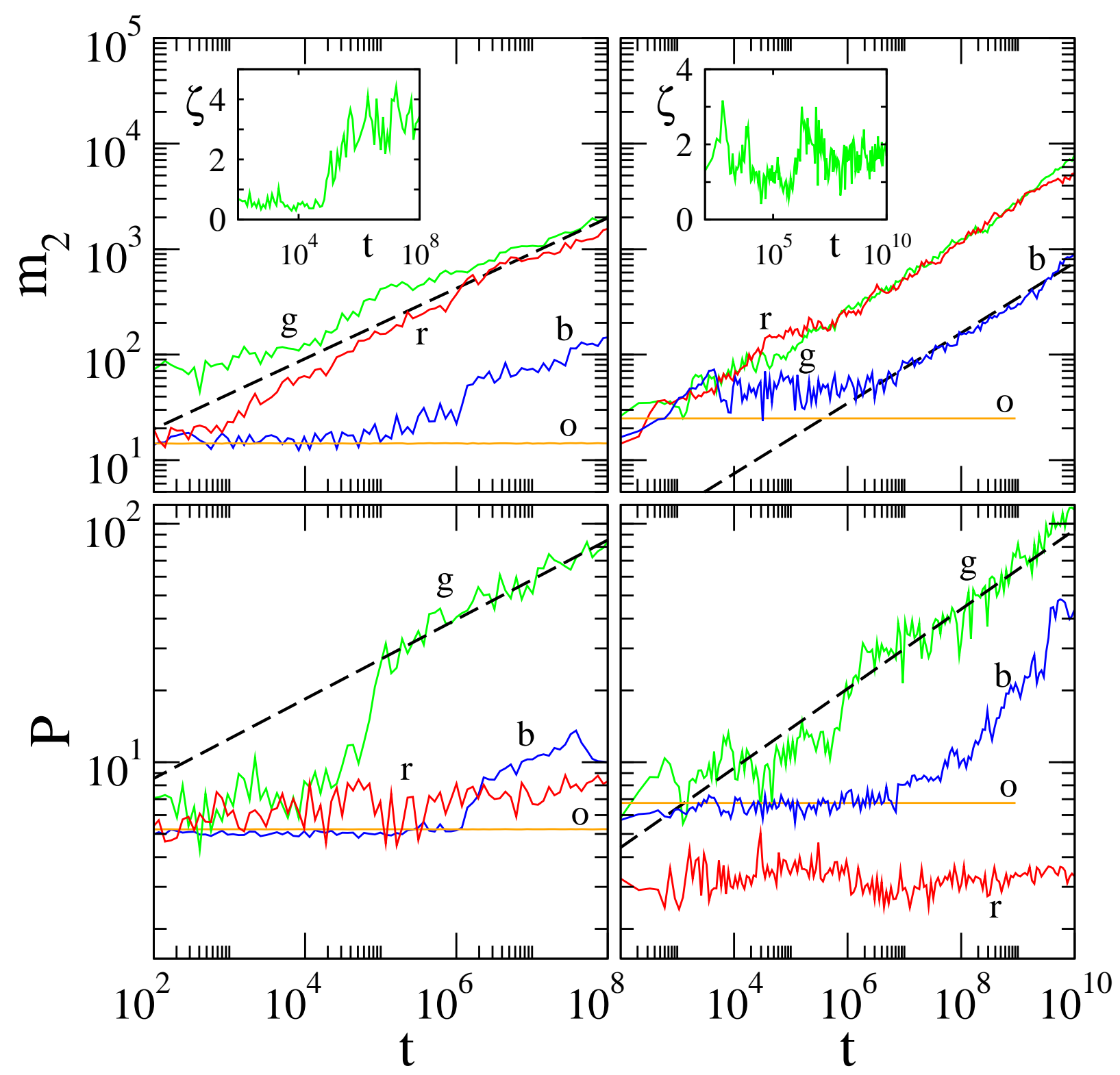

FIG. 2: (color online) Single site excitations. $m_{2}$ and $P$ versus time in $\log -\log$ plots. Left plots: DNLS with $W=4, \beta=0,0.1,1,4.5$ [(o), orange; (b), blue; (g) green; (r) red]. Right plots: KG with $W=4$ and initial energy $E=0.05,0.4,1.5$ [(b) blue; (g) green; (r) red]. The orange curves (o) correspond to the solution of the linear equations of motion, where the term $u_{l}^{3}$ in (7) was absent. The disorder realization is kept unchanged for each of the models. Dashed straight lines guide the eye for exponents $1 / 3\left(m_{2}\right)$ and $1 / 6(P)$ respectively. Insets: the compactness index $\zeta$ as a function of time in linear-log plots for $\beta=1$ (DNLS) and $E=0.4$ (KG). 
of the packet, i. e. when only sites 1 and $K(K \gg 1)$ are excited to an amplitude $1 / 2$, the compactness index is $\zeta=16 / K^{2}$. So, smaller values of $\zeta$ correspond to more sparse wave packets.

We expect that $\zeta$ in regime I will remain constant for $t<\tau_{d}$ and will behave as in the case of regime II for latter times. In regime II $\zeta$ would either be constant or decay in time, while in regime III it should decay since $P$ remains practically constant. The time evolution of $\zeta$ for excitations in regime II is shown in the insets of Fig. 2, As one can see the compactness index oscillates around some constant nonzero value both for the DNLS and the KG models. This means that the wave packet spreads but does not become more sparse. For the particular cases of Fig. 2 the compactness index attains the values $\zeta=3.5$ for the DNLS model at $t=10^{8}$ and $\zeta=1.7$ for the KG chain at $t=10^{10}$. The corresponding wave packet of the DNLS model is shown in the left plots of Fig. 3.

Partial nonlinear localization in regime III is explained by selftrapping [10]. It is due to tuning frequencies of excitations out of resonance with the NM spectrum, takes place irrespective of the presence of disorder and is related to the presence of exact $t$-periodic spatially localized states (also coined discrete breathers) for ordered [19] and disordered systems [20] (in the latter case also $t$-quasiperiodic states exist). These exact solutions act as trapping centers.

Note that for large nonlinearities $(\beta \gg \Delta$ for DNLS or large energy values $E$ of the KG model) almost the whole excitation is selftrapped. This behavior can be seen in the left plots of Fig. 4, where the time evolution of $m_{2}$ and $P$ for different values of the energy $\mathrm{E}$ of the KG chain is shown. The value of $W$ is kept to $W=4$ as in the cases presented in the right plots of Fig. 2. As the energy increases the portion of the wave packet that stays selftrapped increases with respect to the part that diffuses. Thus, we observe a change in the evolution of $m_{2}$ from subdiffusive increase to practical constancy. On the other hand, $P$ is not affected as it continues to fluctuate around some constant value.

Anderson localization on finite times in regime I is observed on potentially large time scales $\tau_{d}$, and as in III, regular states act as trapping centers [20]. For $t>\tau_{d}$, the wave packet trajectory finally departs away from the vicinity of regular orbits, with subsequent spreading. Increasing the value of $W$ results to small localization lengths of NMs and thus, Anderson localization will persist for extremely long time intervals. Since our numerical computations are limited in time, we are not able to observe the detrapping phase of the 


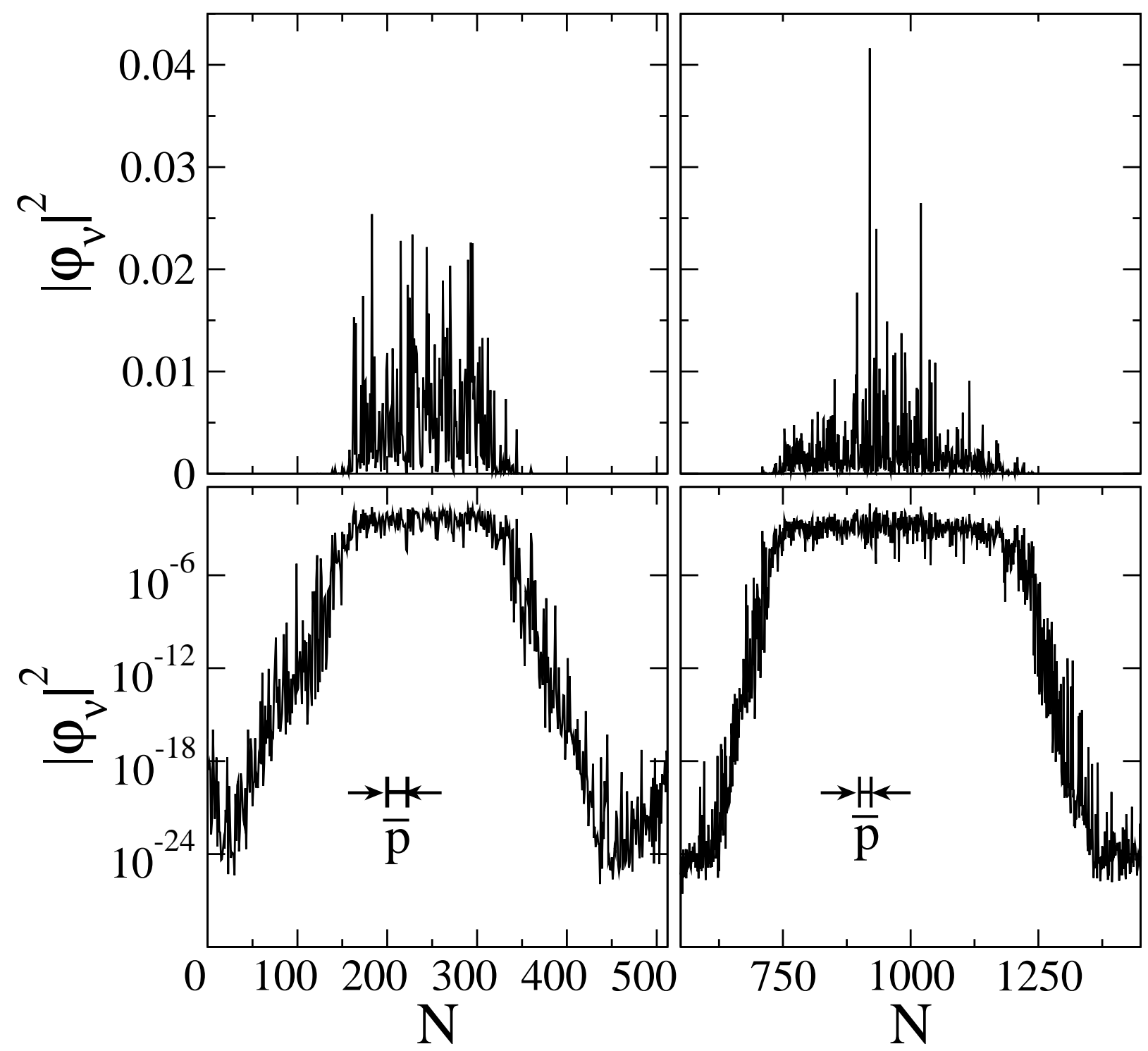

FIG. 3: Norm density distributions in the NM space at time $t=10^{8}$ for the initial excitations in the regime II of the DNLS model shown in the left plots of Figs. 2 and 5. Left plots: single site excitation for $W=4$ and $\beta=1$. Right plots: single mode excitation for $W=4$ and $\beta=5$. $\left|\phi_{\nu}\right|^{2}$ is plotted in linear (logarithmic) scale in the upper (lower) plots. The maximal mean value of the localization volume of the NMs $\bar{p} \approx 22$ (shown schematically in the lower plots) is much smaller than the length over which the wave packets have spread.

evolution when $W$ increases significantly. This behavior can be seen in the right plots of Fig. 4 where we consider initial single site excitations which, for $W=4$ (see right plots of Fig. 2) belong to regime I. In these plots we observe a direct transition from regime I to practical constancy of $m_{2}$ and $P$ as $W$ increases, at least up to the final integration time 


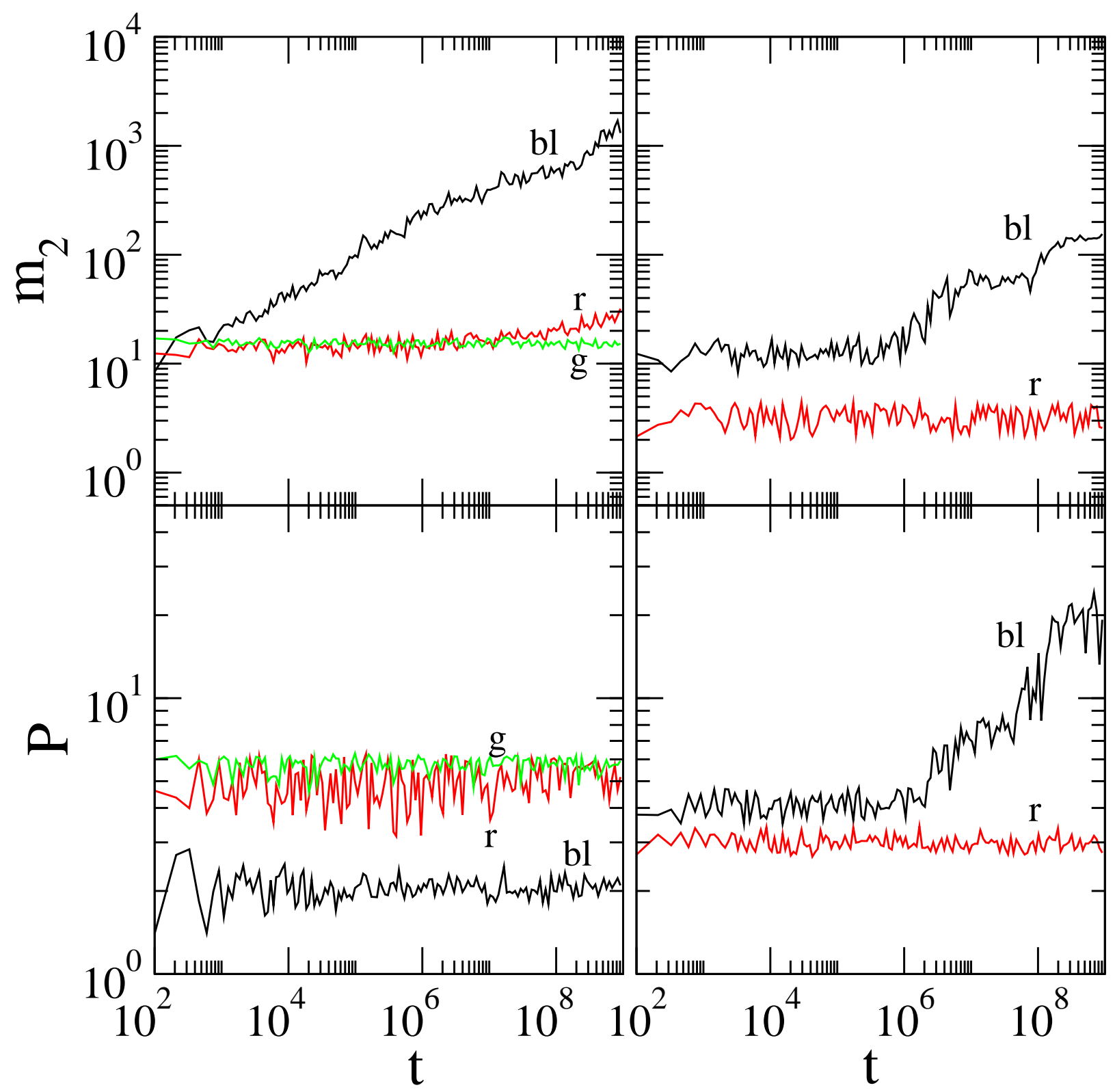

FIG. 4: (color online) Single site excitations for the same disorder realization of the KG model. $m_{2}$ and $P$ versus time in $\log -\log$ plots. Left panels: plots for $W=4$ and initial energy $E=3.225,4,10$ [(bl) black; (r) red; (g) green]. Right panels: Plots for $E=0.05$ and $W=6,7$ [(bl) black; (r) red].

used.

For single mode excitations we find a similar outcome, but with rescaled critical values for the nonlinearity strength which separate the different regimes. Examples of the three different regimes are shown in Fig.5. As in the case of single site excitations presented in Fig. 2, the compactness index $\zeta$ plotted in the insets if Fig. 5 remains practically constant 

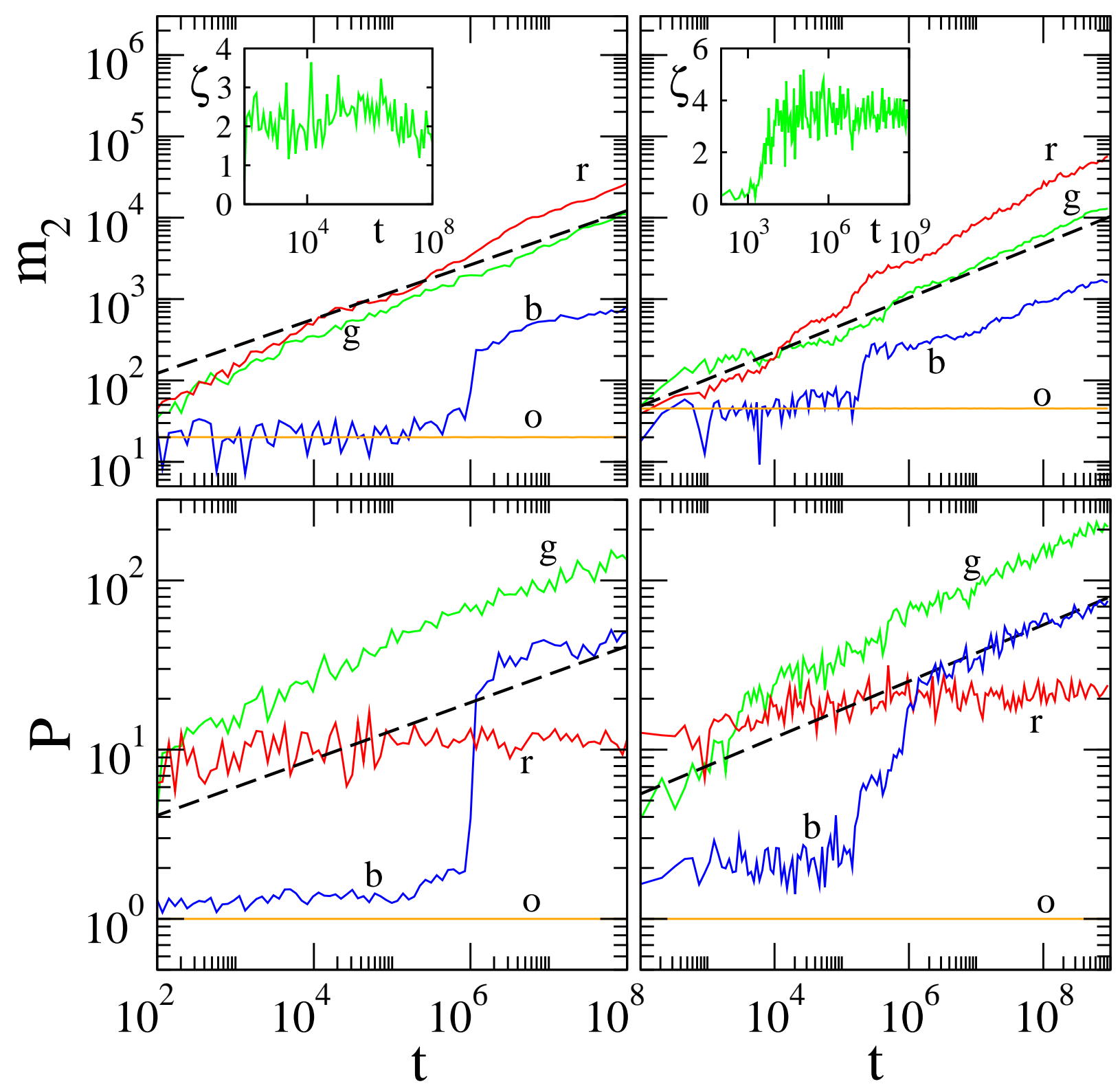

FIG. 5: (color online) Single mode excitations. $m_{2}$ and $P$ versus time in log-log plots. Left plots: DNLS with $W=4, \beta=0,0.6,5,30$ [(o) orange; (b) blue; (g) green; (r) red]. Right plots: KG with $W=4$ and initial energy $E=0.17,1.1,13.4$ [(b) blue; (g) green; (r) red]. The orange curves (o) correspond to the solution of the linear equations of motion, where the term $u_{l}^{3}$ in (7) was absent. The disorder realization is kept unchanged for each of the models. Dashed straight lines guide the eye for exponents $1 / 3\left(m_{2}\right)$ and 1/6 $(P)$ respectively. Insets: the compactness index $\zeta$ as a function of time in linear-log plots for $\beta=5$ (DNLS) and $E=1.1(\mathrm{KG})$. 
for excitations in regime II, attaining the values $\zeta=1.5$ at $t=10^{8}$ for the DNLS model and $\zeta=3.3$ at $t=10^{9}$ for the KG chain. The final norm density distribution for the DNLS model is plotted in the right plots of Fig. 3. The average value $\bar{\zeta}$ of the compactness index over 20 realizations at $t=10^{8}$ for the DNLS model with $W=4$ and $\beta=5$ was found to be $\bar{\zeta}=2.95 \pm 0.39$.

\section{Spreading}

The subdiffusive spreading takes place in regime I for $t>\tau_{d}$, in regime II, and for a part of the wave packet also in regime III. For single site excitations the exponent $\alpha$ does not appear to depend on $\beta$ in the case of the DNLS model or on the value of $E$ in the case of KG. In Fig,6] we show results for $m_{2}(t)$ in regime II for different values of the disorder strength $W$. Again we find no visible dependence of the exponent $\alpha$ on $W$. Therefore the subdiffusive spreading is rather universal and the parameters $\beta$ (or $E$ ) and $W$ are only affecting the

prefactor. Excluding selftrapping, any nonzero nonlinearity will completely delocalize the wave packet and destroy Anderson localization. We performed fittings by analyzing 20 runs in regime II with different disorder realizations. For each realization we fitted the exponent $\alpha$, and then averaged over all computational measurements. We find $\alpha=0.33 \pm 0.02$ for DNLS, and $\alpha=0.33 \pm 0.05$ for KG. Therefore, the predicted universal exponent $\alpha=1 / 3$ [11] appears to explain the data.

On the other hand, in the case of single mode excitations the numerically computed values of the exponent $\alpha$ seem to be slightly larger than $\alpha=1 / 3$, as can be also seen from the results of Fig. 5. In particular, $m_{2}$ in regimes II and III of the DNLS model and in regime III of the KG model increases slightly faster than $\propto t^{1 / 3}$, which is represented by the dashed lines in the upper plots of Fig. 5. In addition, the value of the exponent seems to slightly vary with respect to the nonlinearity parameter $\beta$ for DNLS and $E$ for KG. The reason of the slightly different behavior between single site and single mode excitations is still an open issue. 


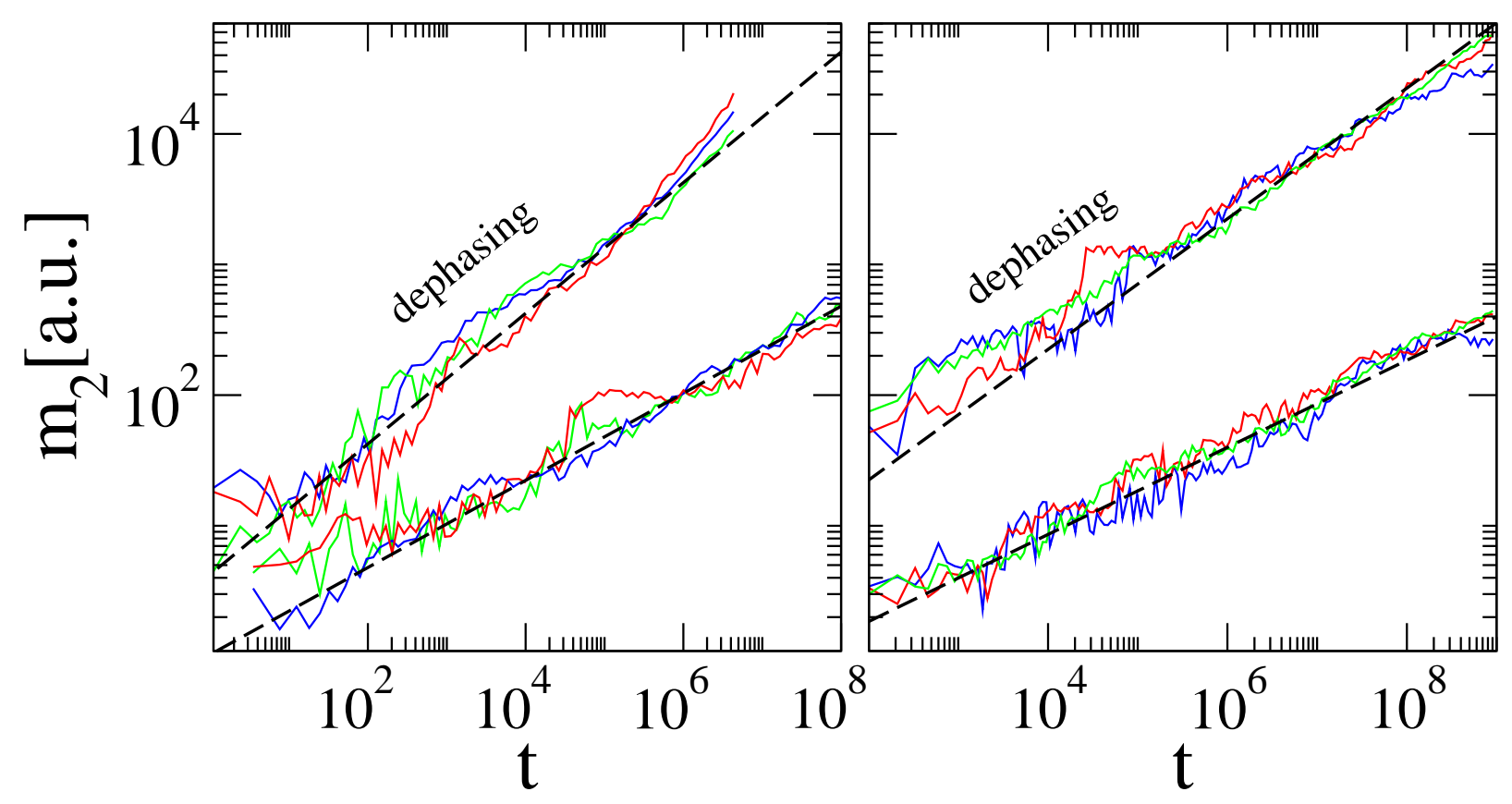

FIG. 6: (color online) Single site excitations. $m_{2}$ (in arbitrary units) versus time in log-log plots in regime II and different values of $W$. Lower set of curves: plain integration (without dephasing); upper set of curves: integration with dephasing of NMs (see section IVA). Dashed straight lines with exponents $1 / 3$ (no dephasing) and $1 / 2$ (dephasing) guide the eye. Left plot: DNLS, $W=4$, $\beta=3$ (blue); $W=7, \beta=4$ (green); $W=10, \beta=6$ (red). Right plot: KG, $W=10, E=0.25$ (blue) , $W=7, E=0.3$ (red) $, W=4, E=0.4$ (green). The curves are shifted vertically in order to give maximum overlap within each group.

\section{E. Detrapping}

In the intermediate regime II the wave packet starts to spread almost from scratch. We do not observe any saturation and crossover into localization on later times. Let us assume that the wave packet spreads without limitations. The initial nonlinear frequency shift $\delta_{l}$ was larger than the average level spacing in a localization volume $\overline{\Delta \lambda}$. However, $\delta_{l}$ will become smaller than $\overline{\Delta \lambda}$ at some later time, since $\sup _{l}\left|\psi_{l}\right|^{2}\left(\sup _{l} E_{l}\right.$ for KG) decreases in time as the wave packet spreads. Therefore, there will be a large but finite time $t_{d}$, at which we cross over from the intermediate regime II into the weak nonlinearity regime I. The arresting of the wave packet up to a time $\tau_{d}$ in the weak nonlinearity regime I can be explained by a correspondingly large spreading time scale $\tau_{d}$. For $t<\tau_{d}$ no spreading is observed when monitoring the second moment $m_{2}$, with subsequent spreading observed on 

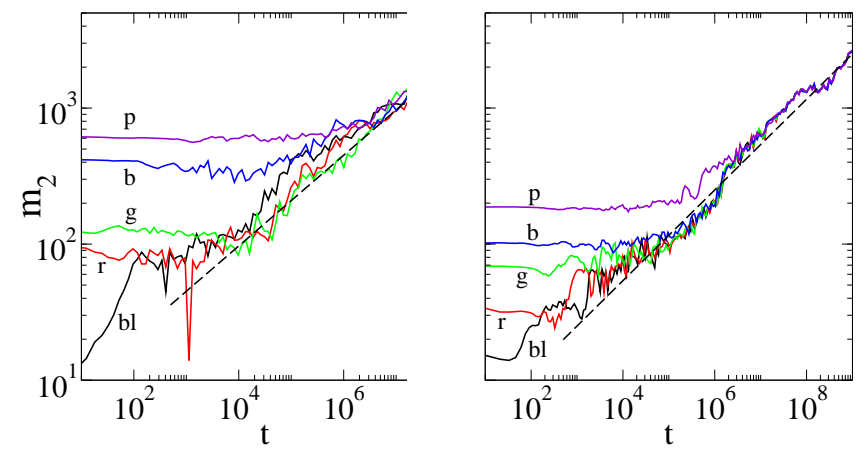

FIG. 7: (color online) Evolution of $m_{2}$ versus time in log-log plots. Single site excitations in the intermediate regime II for the DNLS (left plot) and the KG model (right plot) correspond to black curves (bl). The wave packets after $t_{d}=10^{3}, 10^{4}, 10^{5}, 10^{6}$ time units (t. u.) [(r) red; (g) green; (b) blue; (p) purple] are registered and relaunched as initial distributions (colored curves). The dashed straight lines correspond to functions $\propto t^{1 / 3}$.

larger time scales $t>\tau_{d}$.

We test the above conclusions by the following simple scheme. We start a single site excitation in the intermediate regime II, measure the distribution at some time $t_{d}$, and relaunch the distribution as an initial condition at time $t=0$. The results are shown in Fig.7. We find that the relaunched runs yield a second moment $m_{2}$ which appears to be constant up to the time $\tau_{d} \approx t_{d}$ with a subsequent spreading, similar to the previously obtained detrapping in regime $\mathrm{I}$.

For a specific value of the nonlinearity $\beta$ of the DNLS model let each NM in the packet after some spreading to have norm $\left|\phi_{\nu}\right|^{2} \sim n \ll 1$ with $n$ denoting the average norm density of the excited NMs (in the case of the KG model $n$ corresponds to the average energy density of the excited NMs). The packet size is then $1 / n \gg \bar{p}$, with $\bar{p}=\max _{\nu} \overline{p_{\nu}}$, and the second moment $m_{2} \sim 1 / n^{2}$. Let us assume that the second moment grows as $m_{2} \sim t^{1 / 3}$. Let us also assume, that at any time the spreading is due to some diffusion process, and is characterized by some momentary diffusion rate $D(t)$ such that $m_{2}=D(t) t$. Then it follows that $D(t) \sim t^{-2 / 3}$ and finally $D \sim n^{4}$. Such a result has to be the outcome of the action of the nonlinear terms (which always contain products $\beta n$ ). A diffusion rate is equal to an inverse characteristic time scale, and therefore we conjecture

$$
D=\tau_{d}^{-1} \sim \beta^{4} n^{4}
$$

There are two ways of modifying $D$. We can either spread our initial excitation over some 
number of sites $L$, therefore varying $n$. Alternatively we can fix the shape of the initial excitation, and vary $\beta$.

In order to test the validity of Eq. (17) for a fixed value of nonlinearity we considered a single site excitation in the intermediate regime II for the KG model with total energy $E=0.4$, so that $m_{2}$ and $P$ start to grow from the beginning (black curves in Figs. 8 (a) and (b) respectively). We also followed the time evolution of wave packets having as initial condition a homogeneous distribution of the energy $E=0.4$ among $L$ neighboring sites. In particular, we considered initial distributions with $u_{l}=0$ and $p_{l}=0$ except for the central $L$ sites whose initial momenta were set to $\pm \sqrt{2 E / L}$, with the sign changing randomly from site to site. We performed simulations with $L$ ranging from 1 up to 41 . The time evolution of $m_{2}$ and $P$ for some of these cases is shown in Figs. 8(a) and (b) respectively. In accordance to the results presented in Fig. 7 we observe that, distributing the energy of a single site excitation belonging to regime II over more sites results in a time dependence of $m_{2}$ and $P$ similar to regime I, i. e. both quantities start to increase after some transient detrapping time $\tau_{d}$.

The behavior of the second moment $m_{2}(t)$ can be modeled by a function of the form

$$
m_{2}(t)=M\left(t+\tau_{d}\right)^{\alpha}
$$

where $M$ is a constant related to the value of the second moment of the initial distribution $m_{2}(0)=M \tau_{d}^{\alpha}$. Eq. (18) gives a power law dependence of $m_{2}$ on $t$ for $t \gg \tau_{d}$ and a slow time dependence of $m_{2}$ for $t \ll \tau_{d}$. Thus, it can be used to describe the behavior of $m_{2}$ for $L>1$. Fitting the numerical data obtained for different values of $L$ by Eq. (18) (see Fig. 8(c) for such an example) we can determine the dependence of $\tau_{d}$ on $L$ (Fig. 8(d)). Since $L \sim n^{-1}$ from (17) we conclude that $\tau_{d} \sim L^{4}$. As we can see from Fig. 8(d) the numerically obtained results are in good agreement with this assumption.

To test the dependence of $D$ on $\beta$, we studied the weak nonlinearity regime I for the DNLS model with $W=4$. We launched single site excitations for 10 realizations for $\beta=0.1$ and $\beta=0.2$. We estimated the detrapping times $\tau_{d}$ on logarithmic scale for each run, and averaged over each group of realizations. As a result we obtain $\left\langle\log _{10} \tau_{d}\right\rangle=5$ for $\beta=0.2$, and $\left\langle\log _{10} \tau_{d}\right\rangle=6.9$ for $\beta=0.1$ (with $\langle\cdots\rangle$ denoting the mean value over the realizations), and their difference is then 1.9. According to (17), the difference should be 1.2 which is in relatively good agreement with the numerically estimated value. 


\section{F. Numerical accuracy and roundoff errors}

We performed several tests in order to ensure that our results are not generated by inaccurate computations. First we varied the size of the system and found no dependence of the results on it. Therefore we exclude finite size effects.

Second we varied the time steps of the symplectic integration schemes by orders of magnitudes. Again we found no visible change in the detrapping times, or in the spreading characteristics. We also used different integration schemes, and even nonsymplectic ones (8th order Runge-Kutta). No changes were obtained either. Therefore we exclude effects due to discretization of time.

Finally we studied the influence of computational roundoff errors. The above observation, that the variation of time steps does not change the key results, implicitly tells that roundoff errors can be excluded as well. Indeed, changing the time steps, we change the number of operations to be performed on a given interval of integration. Therefore we change the number of roundoff operations.

In addition, we decided to perform further tests with respect to the roundoff error issue. These tests are inspired by the following consideration. Floating point numbers are characterized by the number of digits $a$ after the comma which are kept during computations. All presented data were obtained with double precision, where $a=16$. The detrapping and spreading can be only due to the cubic nonlinear terms in the equations of motion. These terms are added to linear terms, when calculating the rhs of (2) and (7). Therefore, when for example in the case of the DNLS model $\sup _{l}\left|\psi_{l}\right|^{2}<10^{-8}$, the nonlinear terms become of the order of the roundoff error of the linear terms. For all of our simulations, the amplitudes in the packet are of the order of $10^{-2}$ or larger. Therefore the roundoff is affecting only the amplitudes far in the exponential tails. We changed the calculation to single precision, for which $a=8$, but we did not observe any qualitative difference in our results. For single precision the nonlinear terms will be affected by roundoff errors when $\sup _{l}\left|\psi_{l}\right|^{2}<10^{-4}$, which is still realized only in the exponential tails. We note, that the times at which the roundoff errors affect the packet modes correspond to $t \sim 10^{80}$ for $a=16$ and $t \sim 10^{30}$ for $a=8$ which are obviously not accessible with our computation schemes.

Therefore we implemented a brute force roundoff scheme: after each time step of integration we take the distributions and perform a roundoff at a prescribed digit $a=1,2,3,4, \ldots$. 
We expect therefore to reduce the time at which roundoff errors will become visible, in order to observe that effect within the time window accessible by our computations. Indeed, we find that strong fluctuations in the conserved quantities set in at a time $t_{r}$ which decreases with decreasing $a$. In particular for the DNLS we find $t_{r} \approx 10^{3}, 10^{5}, 10^{7}$ for $a=1,2,3$, and for the KG model we find $t_{r} \approx 10^{3}, 10^{5}, 10^{8}$ for $a=1,2,3$. When monitoring the second moment and the participation number, we also find strong deviations from the above results at times $t>t_{r}$. For $a \geq 4$ we do not observe any significant change in the data. Therefore we conclude, that the roundoff errors with double (or even single) precision are not affecting our results.

\section{SPREADING MECHANISMS}

We can think of two possible mechanisms of wave packet spreading. A NM with index $\mu$ in a layer of width $\bar{p}$ in the cold exterior, which borders the packet, is either incoherently heated by the packet, or resonantly excited by some particular NM from a layer with width $\bar{p}$ inside the packet. Heating here implies a (sub)diffusive spreading of energy. Note that the numerical results yield subdiffusion, supporting the nonballistic diffusive heating mechanism.

For heating to work, the packet modes $\phi_{\nu}(t)$ should contain a part $\phi_{\nu}^{c}(t)$, having a continuous frequency spectrum (similar to a white noise), in addition to a regular part $\phi_{\nu}^{r}(t)$ of pure point frequency spectrum:

$$
\phi_{\nu}(t)=\phi_{\nu}^{r}(t)+\phi_{\nu}^{c}(t)
$$

Therefore at least some NMs of the packet should evolve chaotically in time. The more the packet spreads, the less the mode amplitudes in the packet become. Therefore its dynamics should become more and more regular, implying $\lim _{t \rightarrow \infty} \phi_{\nu}^{c}(t) / \phi_{\nu}^{r}(t) \rightarrow 0$.

\section{A. Are all packet modes chaotic?}

In Ref. [8] it was assumed that all NMs in the packet are chaotic, and their phases can be assumed to be random at all times. At variance to the above expectation, it follows that $\phi_{\nu}^{r}(t)=0$, or at least the ratio $\phi_{\nu}^{c}(t) / \phi_{\nu}^{r}(t)$ is constant on average. Consequently $\left|\phi_{\nu}^{c}(t)\right| \sim n^{1 / 2}$ where $n$ is the average norm density in the packet. 
According to (4) the heating of the exterior mode should evolve as $i \dot{\phi}_{\mu} \approx \lambda_{\mu} \phi_{\mu}+\beta n^{3 / 2} f(t)$ where $\left\langle f(t) f\left(t^{\prime}\right)\right\rangle=\delta\left(t-t^{\prime}\right)$ ensures that $f(t)$ has a continuous frequency spectrum. Then the exterior NM increases its norm according to $\left|\phi_{\mu}\right|^{2} \sim \beta^{2} n^{3} t$. The momentary diffusion rate of the packet is given by the inverse time $T$ it needs to heat the exterior mode up to the packet level: $D=1 / T \sim \beta^{2} n^{2}$. The diffusion equation $m_{2} \sim D t$ yields $m_{2} \sim \beta t^{1 / 2}$. We tested the above conclusions by enforcing decoherence of NM phases. Each 100 time units on average $50 \%$ of the NMs were randomly chosen, and their phases were shifted by $\pi$ (DNLS). For the KG case we changed the signs of the corresponding NM momenta. We obtain $m_{2} \sim t^{1 / 2}$ (see Fig[6). Therefore, when the NMs dephase completely, the exponent $\tilde{\alpha}=1 / 2$, contradicting numerical observations without dephasing. Thus, not all NMs in the packet are chaotic, and dephasing is at best a partial outcome.

\section{B. Mode-mode resonances inside the packet}

Chaos is a combined result of resonances and nonintegrability. Let us estimate the number of resonant modes in the packet for the DNLS model. Excluding secular interactions, the amplitude of a NM with $\left|\phi_{\nu}\right|^{2}=n_{\nu}$ is modified by a triplet of other modes $\vec{\mu} \equiv\left(\mu_{1}, \mu_{2}, \mu_{3}\right)$ in first order in $\beta$ as (4)

$$
\left|\phi_{\nu}^{(1)}\right|=\beta \sqrt{n_{\mu_{1}} n_{\mu_{2}} n_{\mu_{3}}} R_{\nu, \vec{\mu}}^{-1}, R_{\nu, \vec{\mu}} \sim\left|\frac{\overrightarrow{d \lambda}}{I_{\nu, \mu_{1}, \mu_{2}, \mu_{3}}}\right|,
$$

where $\overrightarrow{d \lambda}=\lambda_{\nu}+\lambda_{\mu_{1}}-\lambda_{\mu_{2}}-\lambda_{\mu_{3}}$. The perturbation approach breaks down, and resonances set in, when $\sqrt{n_{\nu}}<\left|\phi_{\nu}^{(1)}\right|$. Since all considered NMs belong to the packet, we assume their norms to be equal to $n$ for what follows. If three of the four mode indices are identical, one is left with interacting NM pairs. A statistical analysis of the probability of resonant interaction was performed in Ref. [11]. For small values of $n$ (i.e. when the packet has spread over many $\mathrm{NMs}$ ) the main contribution to resonances are due to rare multipeak modes [11], with peak distances being larger than the localization volume. If two or none of the four mode indices are identical, one is left with triplets and quadruplets of interacting NMs respectively. In both cases the resonance conditions can be met at arbitrarily small values of $n$ for NMs from one localization volume.

We perform a statistical numerical analysis for the quadruplet case. For a given NM $\nu$ we obtain $R_{\nu, \vec{\mu}_{0}}=\min _{\vec{\mu}} R_{\nu, \vec{\mu}}$. Collecting $R_{\nu, \vec{\mu}_{0}}$ for many $\nu$ and many disorder realizations, 
we find the probability density distribution $\mathcal{W}\left(R_{\nu, \vec{\mu}_{0}}\right)$ (Fig. 9). The main result is that $\mathcal{W}\left(R_{\nu, \vec{\mu}_{0}} \rightarrow 0\right) \rightarrow C(W) \neq 0$. For the cases studied, the constant $C$ drops with increasing disorder strength $W$. Similar results are found if pairs of resonant NMs [11] are analyzed, with the only difference that the constant $C$ is reduced e.g. by a factor of 30 for $W=4$.

The probability $\mathcal{P}$ for a mode, which is excited to a norm $n$ (the average norm density in the packet), to be resonant with at least one triplet of other modes at a given value of the interaction parameter $\beta$ is given by

$$
\mathcal{P}=\int_{0}^{\beta n} \mathcal{W}(x) \mathrm{d} x,
$$

with $x$ denoting $R_{\nu, \vec{\mu}_{0}}$. For $\beta n \ll 1$ it follows

$$
\mathcal{P} \approx C \beta n
$$

Therefore the probability for a mode in the packet to be resonant is proportional to $C \beta n$. On average the number of resonant modes in the packet is constant, proportional to $C \beta$, and their fraction within the packet is $\sim C \beta n$. Since packet mode amplitudes fluctuate in general, averaging is meant both over the packet, and over suitably long time windows (yet short compared to the momentary inverse packet growth rate). We conclude, that the continuous frequency part of the dynamics of a packet mode is scaled down by $C \beta n$, compared to the case when all NMs would be chaotic. It follows that $\phi_{\nu}^{c}(t) / \phi_{\nu}^{r}(t) \sim C \beta n$. As expected initially, the chaotic part in the dynamics of packet modes becomes the weaker the more the packet spreads, and the packet dynamics becomes more and more regular in the limit of large times. Therefore the chaotic component $\phi_{\nu}^{c}(t) \ll \phi_{\nu}^{r}(t)$ is a small parameter.

Expanding the term $\left|\phi_{\nu}\right|^{2} \phi_{\nu}$ to first order in $\phi_{\nu}^{c}(t)$, the heating of the exterior mode should evolve according to $i \dot{\phi}_{\mu} \approx \lambda_{\mu} \phi_{\mu}+C \beta^{2} n^{5 / 2} f(t)$. It follows $\left|\phi_{\mu}\right|^{2} \sim C^{2} \beta^{4} n^{5} t$, and the rate $D=1 / T \sim C^{2} \beta^{4} n^{4}$ (cf. the prediction (17)). The diffusion equation $m_{2} \sim D t$ yields

$$
m_{2} \sim C^{2 / 3} \beta^{4 / 3} t^{\alpha}, \alpha=1 / 3
$$

The predicted exponent $\alpha=1 / 3$ is close to the numerically observed one, as we discussed in section IIID. 


\section{Resonant spreading?}

Finally we consider the process of resonant excitation of an exterior mode by a mode from the packet. The number of packet modes in a layer of the width of the localization

volume at the edge, which are resonant with a cold exterior mode, will be proportional to $\beta n$. After long enough spreading $\beta n \ll 1$. On average there will be no mode inside the packet, which could efficiently resonate with an exterior mode. Therefore, resonant growth can be excluded.

\section{SUMMARY AND DISCUSSION}

We studied the spreading of wave packets in disordered one-dimensional nonlinear chains. In particular we considered two systems, namely the DNLS model (1) and the quartic KG system (66). The linear parts of these two models are equivalent in the sense that they correspond to the same eigenvalue problem (33).

We predicted theoretically and verified numerically the existence of three different dynamical behaviors depending on the relation of the nonlinear frequency shift $\delta$ (which is proportional to the system's nonlinearity) with the average spacing $\overline{\Delta \lambda}$ of eigenfrequencies and the spectrum width $\Delta(\overline{\Delta \lambda} \leq \Delta)$ of the linear system. The dynamics for small nonlinearities $(\delta<\overline{\Delta \lambda})$ is characterized by localization as a transient, with subsequent subdiffusion (regime I). For intermediate values of the nonlinearity $\overline{\Delta \lambda}<\delta<\Delta$, and the wave packets exhibit immediate subdiffusion (regime II). In this case, the second moment $m_{2}$ and the participation number $P$ increase in time following the power laws $m_{2} \sim t^{\alpha}, P \sim t^{\alpha / 2}$. Assuming that the spreading is due to an incoherent excitation of the cold exterior, induced by the chaotic behavior of the wave packet, we predicted $\alpha=1 / 3$. Finally, for even higher nonlinearities $(\delta>\Delta)$ a large part of the wave packet is selftrapped, while the rest subdiffuses (regime III). In this case $P$ remains practically constant, while $m_{2} \sim t^{\alpha}$. The overall picture is schematically presented in Fig. 1 both for the DNLS and the KG model.

The compactness index $\zeta=P^{2} / m_{2}$, which measures the sparseness of wave packets, exhibits different behaviors for the three dynamical regimes. In particular, the behavior of $\zeta$ for wave packets in regime II imply that these wave packets spread but do not become more sparse. 
For large values of the disorder strength $W$ and/or strong nonlinearity the intermediate regime II effectively disappears, and the evolution will start either in regime I, or in regime III. In regime I the detrapping times increase with further increase of $W$. In regime III the fraction of the wave packet which spreads decreases with increasing nonlinearity. Therefore, large values of $W$ and/or nonlinearity will not allow for an observation of the destruction of Anderson localization on time scales which are bounded from above by practical computational limitations.

The subdiffusive spreading is universal, i. e. the exponent $\alpha$ is independent of the nonlinearity's strength ( $\beta$ for the DNLS model and energy $E$ for the KG one) and $W$, which are only affecting the prefactor in (23). Excluding selftrapping, any nonzero nonlinearity strength $\beta$ will completely delocalize the wave packet and destroy Anderson localization. The exponent $\alpha$ is determined solely by the degree of nonlinearity, which defines the type of overlap integral to be considered in (201), and by the stiffness of the spectrum $\left\{\lambda_{\nu}\right\}$. Our numerical computations confirmed the prediction $\alpha=1 / 3$ in the case of single site and of nonlocal homogeneous excitation. In the case of single mode excitations the three different regimes were also detected. The numerically computed exponents $\alpha$ get slightly larger values than $1 / 3$, exhibiting also a small dependence on the strength of nonlinearity. This discrepancy between the two cases in not clearly understood.

We studied the statistics of detrapping times $\tau_{d}$ for regime I. We provided numerical evidences for the validity of the conjectured dependence of $\tau_{d}$ on the nonlinearity strength and on the average norm density of the excited NMs given in Eq. (17). It is worth mentioning that, distributing the energy of a single site excitation belonging to regime II over more sites results in a time dependence of $m_{2}$ and $P$ similar to regime $I$. In addition, considering as initial condition the profile of a single site excitation in regime II at some latter time $t_{d}$, we observe a dynamical evolution of the type of regime I where the detrapping time is $\tau_{d} \approx t_{d}$.

The spreading of the wave packet is due to weak but nonzero chaotic dynamics inside the packet. It is natural to expect such a dynamics, since the considered systems are nonintegrable. If instead an integrable system is considered, Anderson localization will not be destroyed. Indeed, consider a Hamiltonian in NM representation using actions $J_{\nu}$ and angles $\theta_{\nu}$ as coordinates:

$$
\mathcal{H}_{\text {int }}=\sum_{\nu} \lambda_{\nu} J_{\nu}+\beta \sum_{\nu_{1}, \nu_{2}, \nu_{3}, \nu_{4}} I_{\nu_{1}, \nu_{2}, \nu_{3}, \nu_{4}} \sqrt{J_{\nu_{1}} J_{\nu_{1}} J_{\nu_{1}} J_{\nu_{1}}} .
$$


We assume that the set of eigenfrequencies $\left\{\lambda_{\nu}\right\}$ and the overlap integrals $I_{\nu_{1}, \nu_{2}, \nu_{3}, \nu_{4}}$ are identical with those describing the DNLS model (4), (5). The equations of motion $\dot{J}_{\nu}=$ $-\partial \mathcal{H}_{i n t} / \partial \theta_{\nu}$ and $\dot{\theta}_{\nu}=\partial \mathcal{H}_{i n t} / \partial J_{\nu}$ yield $\dot{J}_{\nu}=0$ since the integrable Hamiltonian (24) depends only on the actions. Therefore, any localized initial condition (e. g. $\left.J_{\nu}(t=0) \propto \delta_{\nu, \nu_{0}}\right)$ will stay localized, since actions of modes which are at large distances will never get excited. Thus, the observed spreading of wave packets, which we studied in detail in the present work, is entirely due to the nonintegrability of the considered models, at variance to (24).

The more the wave packet spreads, the weaker the resonances become. Corresponding structures (chaotic layers) in phase space become thinner and thinner. Consider quantum many-body systems. Classical phase space structures which are finer than the action quantization induced grid become irrelevant. Therefore we may speculate, that the wave packet will stop spreading for a quantum many-body system at some point for zero temperature, but also for temperatures below some finite threshold. These expectations are very close to rigorous results for interacting fermions in disordered systems [22].

In our study we considered initial conditions exciting NMs whose eigenvalues are located close to the center of the frequency band. Thus, the evolution of the system does not significantly depend on the sign of nonlinearity. In contrast, when one excites eigenstates with frequencies near the band edges, a rather weak nonlinearity might lead either to selftrapping or to the weak nonlinear regime depending on the sign of nonlinearity. Such examples were presented in [4] where NMs close to the edges of the band exhibit different dynamical behaviors, i. e. one becomes more localized as the nonlinearity was switched on, while the other tends to delocalize. If a spatially continuous system is considered, then a proper choice of the sign of nonlinearity prohibits selftrapping (so-called defocusing nonlinearity, corresponding to repulsive two-body interactions). For such a case, regime III ceases to exist, and localization is expected to be destroyed irrespectively of the strength of nonlinearity.

\section{Acknowledgments}

We thank B. L. Altshuler, S. Aubry, G. Kopidakis and R. Schilling for useful discussions. 


\section{Appendix A: The $\mathrm{SABA}_{2}$ and $\mathrm{SBAB}_{2}$ symplectic integrators}

In [18] a family of symplectic integrators which involve only forward integration steps was proposed. These integrators were adapted for integrations of perturbed Hamiltonians of the form

$$
H=A+\epsilon B
$$

where both $A$ and $B$ are integrable and $\epsilon$ is a parameter. We briefly recall here their main properties focusing our attention on two particular members of the family of integrators presented in [18], namely the $\mathrm{SABA}_{2}$ and $\mathrm{SBAB}_{2}$ integrators. These integrators have already proved to be very efficient for the numerical study of astronomical [18], as well as accelerator models [21].

Consider a Hamiltonian system of $N$ degrees of freedom having a Hamiltonian $H(\vec{p}, \vec{u})$, with $\vec{p}=\left(p_{1}, \ldots, p_{N}\right), \vec{u}=\left(u_{1}, \ldots, u_{N}\right)$ where $u_{l}$ and $p_{l}, l=1, \ldots, N$, are the generalized coordinates and momenta respectively. An orbit of this system is defined by a vector $\vec{x}(t)=$ $\left(x_{1}(t), \ldots, x_{2 N}(t)\right)$, with $x_{l}=p_{l}, x_{l+N}=u_{l}, l=1, \ldots, N$. This orbit is a solution of Hamilton's equations of motion:

$$
\frac{d \overrightarrow{p_{l}}}{d t}=-\frac{\partial H}{\partial \overrightarrow{u_{l}}}, \frac{d \overrightarrow{u_{l}}}{d t}=\frac{\partial H}{\partial \overrightarrow{p_{l}}}, l=1, \ldots, N,
$$

where $t$ is the independent variable, namely the time. Defining the Poisson bracket of functions $f(\vec{p}, \vec{u}), g(\vec{p}, \vec{u})$ by:

$$
\{f, g\}=\sum_{l=1}^{N}\left(\frac{\partial f}{\partial p_{l}} \frac{\partial g}{\partial u_{l}}-\frac{\partial f}{\partial u_{l}} \frac{\partial g}{\partial p_{l}}\right),
$$

the Hamilton's equations of motion take the form:

$$
\frac{d \vec{x}}{d t}=\{H, \vec{x}\}=L_{H} \vec{x}
$$

where $L_{H}$ is the differential operator defined by $L_{\chi} f=\{\chi, f\}$. The solution of Eq. (A4), for initial conditions $\vec{x}(0)=\vec{x}_{0}$, is formally written as:

$$
\vec{x}(t)=\sum_{n \geq 0} \frac{t^{n}}{n !} L_{H}^{n} \vec{x}_{0}=e^{t L_{H}} \vec{x}_{0} .
$$

A symplectic scheme for integrating (A4) from time $t$ to time $t+\tau$ consists of approximating, in a symplectic way, the operator $e^{\tau L_{H}}=e^{\tau\left(L_{A}+L_{\epsilon B}\right)}$ by an integrator of $j$ steps 
involving products of $e^{c_{i} \tau L_{A}}$ and $e^{d_{i} \tau L_{\epsilon B}}, i=1,2, \ldots, j$, which are exact integrations over times $c_{i} \tau$ and $d_{i} \tau$ of the integrable Hamiltonians $A$ and $B$. The constants $c_{i}, d_{i}$, are chosen so that to increase the order of the remainder of this approximation.

For the $\mathrm{SABA}_{2}$ integrator we get:

$$
\mathrm{SABA}_{2}=e^{c_{1} \tau L_{A}} e^{d_{1} \tau L_{\epsilon B}} e^{c_{2} \tau L_{A}} e^{d_{1} \tau L_{\epsilon B}} e^{c_{1} \tau L_{A}},
$$

with $c_{1}=\frac{1}{2}\left(1-\frac{1}{\sqrt{3}}\right), c_{2}=\frac{1}{\sqrt{3}}, d_{1}=\frac{1}{2}$, while the $\mathrm{SBAB}_{2}$ integrator is given by

$$
\mathrm{SBAB}_{2}=e^{d_{1} \tau L_{\epsilon B}} e^{c_{2} \tau L_{A}} e^{d_{2} \tau L_{\epsilon B}} e^{c_{2} \tau L_{A}} e^{d_{1} \tau L_{\epsilon B}},
$$

with $c_{2}=\frac{1}{2}, d_{1}=\frac{1}{6}, d_{2}=\frac{2}{3}$. Using these integrators we are actually approximating the dynamical behavior of the real Hamiltonian $A+\epsilon B$ by a Hamiltonian $\widetilde{H}=A+\epsilon B+\mathcal{O}\left(\tau^{4} \epsilon+\right.$ $\left.\tau^{2} \epsilon^{2}\right)$, i. e. we introduce an error term of the order $\tau^{4} \epsilon+\tau^{2} \epsilon^{2}$.

The accuracy of the $\mathrm{SABA}_{2}$ (or $\mathrm{SBAB}_{2}$ ) integrator can be improved when the term $C=\{\{A, B\}, B\}$ leads to an integrable system, as in the common situation of $A$ being quadratic in momenta $\vec{p}$ and $B$ depending only on positions $\vec{u}$. In this case, two corrector terms of small backward steps can be added to the integrator $\mathrm{SABA}_{2}$

$$
\mathrm{SABA}_{2} \mathrm{C}=e^{-\tau^{3} \epsilon^{2} \frac{g}{2} L_{C}}\left(\mathrm{SABA}_{2}\right) e^{-\tau^{3} \epsilon^{2} \frac{g}{2} L_{C}} .
$$

A similar expression is valid also for $\mathrm{SBAB}_{2}$. The value of $g$ was chosen in order to eliminate the $\tau^{2} \epsilon^{2}$ dependence of the remainder which becomes of order $\mathcal{O}\left(\tau^{4} \epsilon+\tau^{4} \epsilon^{2}\right)$. In particular we have $g=(2-\sqrt{3}) / 24$ for $\mathrm{SABA}_{2}$ and $g=\frac{1}{72}$ for $\mathrm{SBAB}_{2}$. We note that the $\mathrm{SABA}_{2}$ and $\mathrm{SBAB}_{2}$ integrators involve only forward steps which increases their numerical stability, while, the addition of the corrector results to better accuracy of the schemes, introducing simultaneously a small backward step.

\section{Integration of the KG lattice}

Hamiltonian (6) is suitable for the implementation of the $\mathrm{SABA}_{2} \mathrm{C}$ integration scheme since it attains the form (A1) with:

$$
\begin{aligned}
A & \equiv \sum_{l=1}^{N} \frac{p_{l}^{2}}{2} \\
B & \equiv \sum_{l=0}^{N} \frac{\tilde{\epsilon}_{l}}{2} u_{l}^{2}+\frac{1}{4} u_{l}^{4}+\frac{1}{2 W}\left(u_{l+1}-u_{l}\right)^{2}, \\
\epsilon & =1,
\end{aligned}
$$


where $N$ is the number of anharmonic oscillators. The operators $e^{\tau L_{A}}, e^{\tau L_{B}}, e^{\tau L_{C}}$, which propagate the set of initial conditions $\left(u_{l}, p_{l}\right)$ at time $t$, to their final values $\left(u_{l}^{\prime}, p_{l}^{\prime}\right)$ at time $t+\tau, l=1,2, \ldots, N$ are:

$$
\begin{aligned}
& e^{\tau L_{A}}:\left\{\begin{array}{l}
u_{l}^{\prime}=p_{l} \tau+u_{l} \\
p_{l}^{\prime}=p_{l}
\end{array},\right. \\
& e^{\tau L_{B}}:\left\{\begin{array}{rl}
u_{l}^{\prime} & =u_{l} \\
p_{l}^{\prime} & =\left[-u_{l}\left(\tilde{\epsilon}_{l}+u_{l}^{2}\right)+\frac{1}{W}\left(u_{l-1}+u_{l+1}-2 u_{l}\right)\right] \tau+p_{l}
\end{array},\right. \\
& e^{\tau L_{C}}:\left\{\begin{aligned}
u_{l}^{\prime}= & u_{l} \\
p_{1}^{\prime}= & 2\left\{\left(\frac{2}{W}+\tilde{\epsilon}_{1}+3 u_{1}^{2}\right)\left[-u_{1}\left(\tilde{\epsilon}_{1}+u_{1}^{2}\right)+\frac{1}{W}\left(u_{2}-2 u_{1}\right)\right]\right. \\
& \left.+\frac{1}{W}\left[u_{2}\left(\tilde{\epsilon}_{2}+u_{2}^{2}\right)-\frac{1}{W}\left(u_{3}+u_{1}-2 u_{2}\right)\right]\right\} \tau+p_{1} \\
p_{l}^{\prime}= & 2\left\{\frac{1}{W}\left[u_{l-1}\left(\tilde{\epsilon}_{l-1}+u_{l-1}^{2}\right)-\frac{1}{W}\left(u_{l-2}+u_{l}-2 u_{l-1}\right)\right]\right. \\
& \left.+\frac{2}{W}+\tilde{\epsilon}_{l}+3 u_{l}^{2}\right]\left[-u_{l}\left(\tilde{\epsilon}_{l}+u_{l}^{2}\right)+\frac{1}{W}\left(u_{l-1}+u_{l+1}-2 u_{l}\right)\right] \\
& \left.+\frac{1}{W}\left[u_{l+1}\left(\tilde{\epsilon}_{l+1}+u_{l+1}^{2}\right)-\frac{1}{W}\left(u_{l+2}+u_{l}-2 u_{l+1}\right)\right]\right\} \tau+p_{l}, \quad \text { for } l= \\
p_{N}^{\prime}= & 2\left\{\frac{1}{W}\left[u_{N-1}\left(\tilde{\epsilon}_{N-1}+u_{N-1}^{2}\right)-\frac{1}{W}\left(u_{N-2}+u_{N}-2 u_{N-1}\right)\right]\right. \\
& \left.+\left(\frac{2}{W}+\tilde{\epsilon}_{N}+3 u_{N}^{2}\right)\left[-u_{N}\left(\tilde{\epsilon}_{N}+u_{N}^{2}\right)+\frac{1}{W}\left(u_{N-1}-2 u_{N}\right)\right]\right\} \tau+p_{N}
\end{aligned}\right.
\end{aligned}
$$

since

$$
C=\sum_{l=1}^{N}\left[u_{l}\left(\tilde{\epsilon}_{1}+u_{l}^{2}\right)-\frac{1}{W}\left(u_{l-1}+u_{l+1}-2 u_{l}\right)\right]^{2},
$$

and $u_{0}=u_{N+1} \equiv 0$.

\section{Integration of the DNLS lattice}

We use the $\mathrm{SBAB}_{2}$ integrator scheme to integrate the equations of motion (2), by splitting the DNLS Hamiltonian (1) as

$$
\begin{aligned}
A & \equiv-\sum_{l=1}^{N}\left(\psi_{l+1} \psi_{l}^{*}+\psi_{l+1}^{*} \psi_{l}\right), \\
B & \equiv \sum_{l=1}^{N} \epsilon_{l}\left|\psi_{l}\right|^{2}+\frac{\beta}{2}\left|\psi_{l}\right|^{4}, \\
\epsilon & =1
\end{aligned}
$$


with $N$ being the number of lattice sites. The action of the operator $e^{\tau L_{A}}$ on $\psi_{l}, l=$ $1,2, \ldots, N$ at time $t$ leads to the computation of $\psi_{l}^{\prime}$ at time $t+\tau$, and includes three steps: a) the transformation of the wavefunction from the real $\left(\psi_{l}\right)$ to the Fourier $\left(\varphi_{q}\right)$ space, through a Fast Fourier transform (FFT), b) a rotation of $\varphi_{q}$, and c) the inverse FFT of the wavefunction $\varphi_{q}^{\prime}$ evaluated at the previous step, i. e.

$$
e^{\tau L_{A}}:\left\{\begin{array}{l}
\varphi_{q}=\sum_{m=1}^{N} \psi_{m} e^{2 \pi i q(m-1) / N} \\
\varphi_{q}^{\prime}=\varphi_{q} e^{2 i \cos (2 \pi(q-1) / N) \tau} \\
\psi_{l}^{\prime}=\frac{1}{N} \sum_{q=1}^{N} \varphi_{q}^{\prime} e^{-2 \pi i l(q-1) / N}
\end{array}\right.
$$

On the other hand, the action of $e^{\tau L_{B}}$ on $\psi_{l}$ reduces to a simple rotation in real space, namely

$$
e^{\tau L_{B}}:\left\{\psi_{l}^{\prime}=\psi_{l} e^{-i\left(\epsilon_{l}+\beta\left|\psi_{l}\right|^{2}\right) \tau} .\right.
$$

Note that for the DNLS model we do not apply the two corrector steps since the term $C=\{\{A, B\}, B\}$ does not lead to an easily solvable system.

[1] P. W. Anderson, Phys. Rev. 1091492 (1958).

[2] S. A. Gredeskul and Yu. S. Kivshar, Phys. Rep. 2161 (1992).

[3] T. Schwartz, G. Bartal1, S. Fishman and M. Segev, Nature 44652 (2007)

[4] Y. Lahini, A. Avidan, F. Pozzi, M. Sorel, R. Morandotti, D. N. Christodoulides and Y. Silberberg, Phys. Rev. Lett. 100013906 (2008).

[5] D. Clement A. F. Varon, J. A. Retter, L. Sanchez-Palencia, A. Aspect and P. Bouyer, New J. Phys. 8165 (2006); L. Sanches-Palencia D. Clement, P. Lugan, P. Bouyer, G. V. Shlyapnikov and A. Aspect, Phys. Rev. Lett. 98210401 (2007); J. Billy, V. Josse, Z. Zuo, A. Bernard, 
B. Hambrecht, P. Lugan, D. Clement, L. Sanchez-Palencia, P. Bouyer and A. Aspect, Nature 453, 891 (2008); G. Roati, C. D'Errico, L. Fallani, M. Fattori, C. Fort, M. Zaccanti, G. Modugno, M. Modugno and M. Inguscio, Nature 453, 895 (2008).

[6] D. L. Shepelyansky, Phys. Rev. Lett. 701787 (1993).

[7] M. I. Molina, Phys. Rev. B 5812547 (1998).

[8] A. S. Pikovsky and D. L. Shepelyansky, Phys. Rev. Lett. 100094101 (2008).

[9] I. García-Mata and D. L. Shepelyansky, Phys. Rev. E, 79026205 (2009).

[10] G. Kopidakis, S. Komineas, S. Flach and S. Aubry, Phys. Rev. Lett. 100084103 (2008).

[11] S. Flach, D. Krimer and Ch. Skokos, Phys. Rev. Lett. 102024101 (2009).

[12] O. Morsch and M. Oberthaler, Rep. Prog. Phys. 78, 179 (2006).

[13] Yu. S. Kivshar and G. P. Agrawal, Optical Solitons:From Fibers to Photonic Crystals, Academic Press (Amsterdam), 2003.

[14] B. Kramer and A. MacKinnon, Rep. Prog. Phys. 561469 (1993).

[15] A. D. Mirlin, Phys. Rep. 326259 (2000).

[16] A. A. Ovchinnikov, N. S. Erikhman and K. A. Pronin, Vibrational-Rotational Excitations in Nonlinear Molecular Systems, Kluwer Academic / Plenum Publishers (New York) 2001.

[17] Yu. S. Kivshar and M. Peyrard, Phys. Rev. A 46, 3198 (1992); Yu. S. Kivshar, Phys. Lett. A 173, 172 (1993); M. Johansson, Physica D 216, 62 (2006).

[18] J. Laskar and P. Robutel, Cel. Mech. Dyn. Astr. 8039 (2001)

[19] S. Flach and C. R. Willis, Phys. Rep. 295181 (1998); S. Flach and A. V. Gorbach, ibid. 467, 1 (2008).

[20] C. Albanese and J. Fröhlich, Comm. Math. Phys. 138193 (1991); G. Kopidakis and S. Aubry, Phys. Rev. Lett. 843236 (2000); Id., Physica D 130155 (1999); Id., Physica D 139 247 (2000); J. Bourgain and W.-M. Wang, J. Eur. Math. Soc. 101 (2008); N. K. Efremidis and K. Hizanidis, Phys. Rev. Lett. 101143903 (2008).

[21] L. Nadolski, Ph. D. Thesis, Univ. Paris XI, (2001); L. Nadolski and J. Laskar, Proceedings of the European Particle Accelerator Conference EPAC02, 1276, (2002); Ch. Skokos and Y. Papaphilippou, Proceedings of the European Particle Accelerator Conference EPAC08, $682,(2008)$

[22] D. M. Basko, I. L. Aleiner and B. L. Altshuler, Ann. Phys. (N.Y.) 3211126 (2006).

[23] T. Paul et al., Phys. Rev. A 72063621 (2005); A. Dhar and J. L. Lebowitz, Phys. Rev. Lett. 
100134301 (2008). 

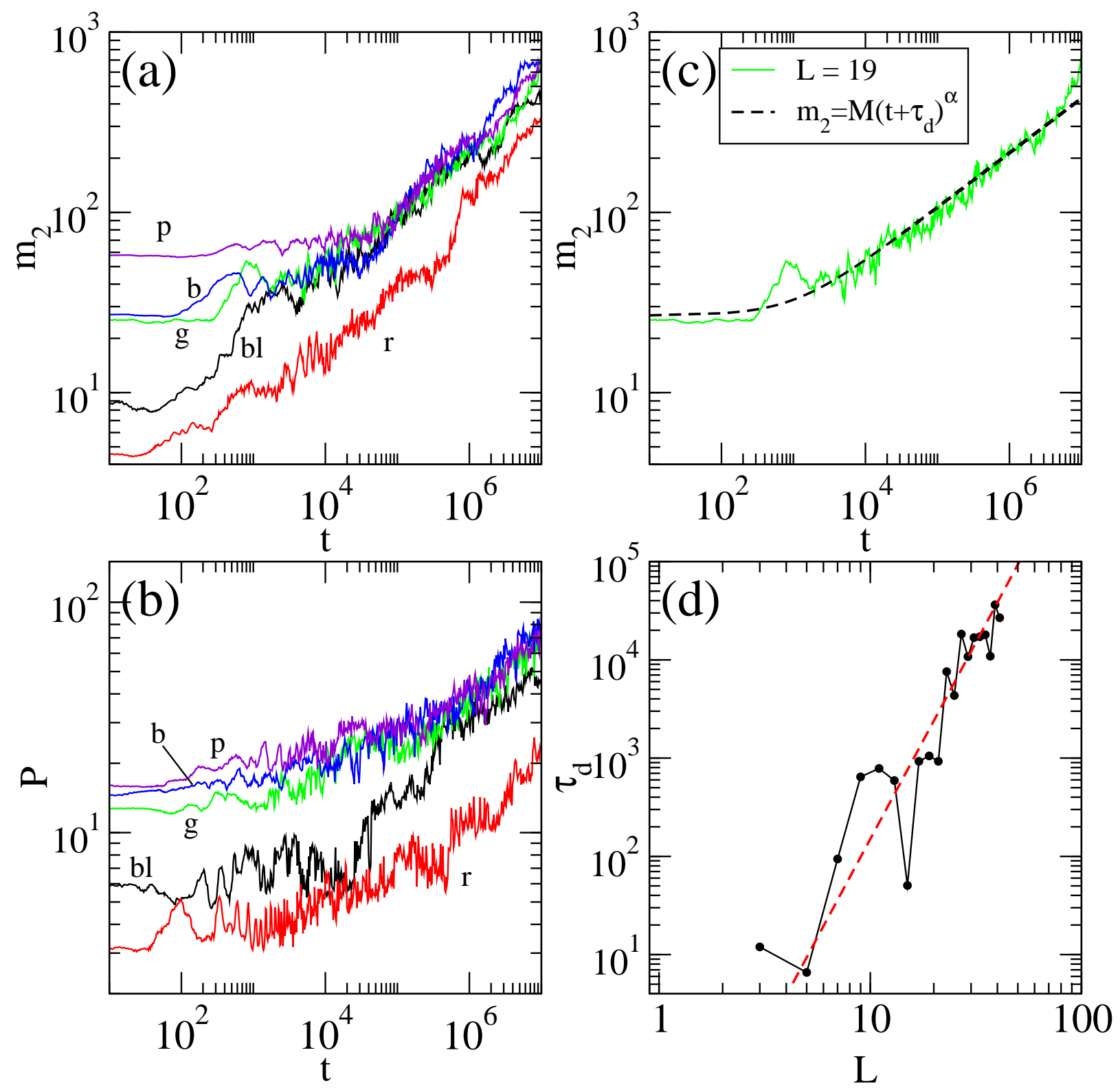

FIG. 8: (color online) Nonlocal excitations of the KG chain corresponding to initial homogeneous distributions of energy $E=0.4$ over $L$ neighboring sites. (a) $m_{2}$ and (b) $P$ versus time in $\log -\log$ plots for $L=1,9,19,29$ and 39 sites [(bl) black; (r) red; (g) green; (b) blue; (p) purple]. (c) Fitting of the time evolution of $m_{2}$ for $L=19$ with a curve of the form (18) for $M=3.25, \tau_{d}=1052$ and $\alpha=0.303$. (d) The dependence of the detrapping time $\tau_{d}$ on the number $L$ of initially excited sites in $\log -\log$ scale. The dashed straight line corresponds to a function $\propto L^{4}$. 


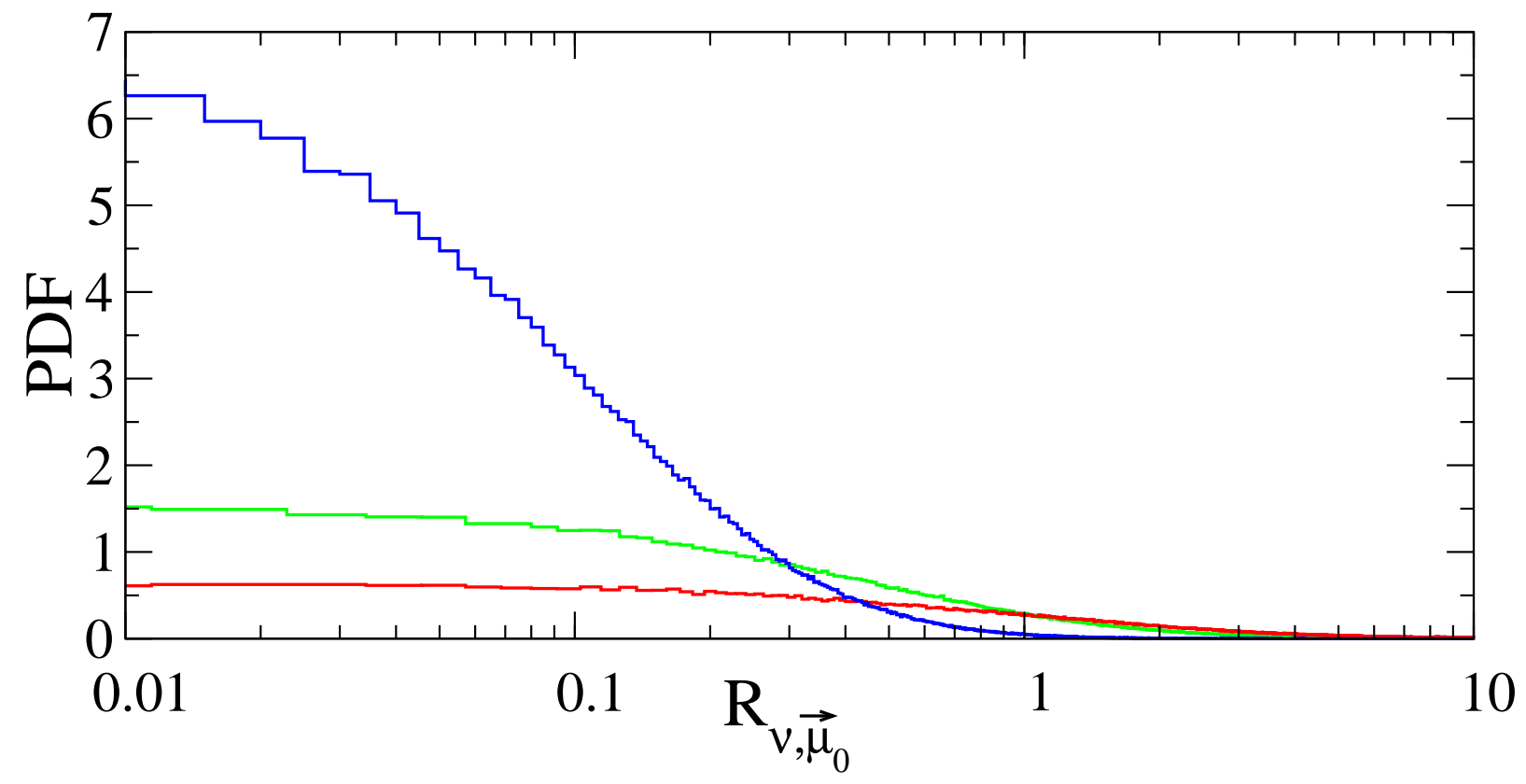

FIG. 9: (color online) Statistical properties of NMs of the DNLS model. Probability densities $\mathcal{W}\left(R_{\nu, \vec{\mu}_{0}}\right)$ of NMs being resonant (see section IVB for details). Disorder strength $W=4,7,10$ (from top to bottom). 